\title{
Research Paper: Ambulatory and Social Performance Status After Transfemoral Prosthetic Rehabilitation Regarding Age, Gender, and Marital Status
}

\author{
Shafiq ur Rehman' ${ }^{1}$, Aqeel Ahmed Khan' ${ }^{1}$, Muhammad Kamran ${ }^{1}$ (D), Ghulam Saqulain ${ }^{2}$ (i), Hilal Ahmed ${ }^{2 *}$ (D) \\ 1. Isra Institute of Rehabilitation Sciences, Isra University, Islamabad, Pakistan. \\ 2. Department of Otorhinolaryngology, Capital Hospital PGMI, Shaheed Zulfiqar Ali Bhutto University, Islamabad, Pakistan.
}

\begin{tabular}{|l|l|l}
\hline $\begin{array}{c}\text { Use your device to scan } \\
\text { and read the article online }\end{array}$ & $\begin{array}{l}\text { Cftation Rehman SU, Khan AA, Kamran M, Saqulain G, Ahmed H. Ambulatory and Social Performance Status After Trans- } \\
\text { femoral Prosthetic Rehabilitation Regarding Age, Gender, and Marital Status. Iranian Rehabilitation Journal. 2021; 19(4):369- }\end{array}$ \\
378. http://dx.doi.org/10.32598/irj.19.4.1335.4
\end{tabular}

Article info:

Received: 07 Jun 2021

Accepted: 07 Aug 2021

Available Online: 01 Dec 2021

\section{Keywords:}

Amputation, Functional status, Lower extremity, Mobility, Prosthesis, Quality of Life

\section{ABSTRACT}

Objectives: This study aims to determine the association of ambulatory and social performance status of transfemoral prosthetic users with their age, gender, and marital status.

Methods: A cross-sectional study was conducted on 400 transfemoral prosthesis users. A sample was recruited from both genders aged 10-60 years using the prosthesis for at least one year. They were selected using the non-probability convenience sampling method from the Pakistan Institute of Prosthetic and Orthotic Sciences from July 2019 to December 2019. Lower extremity functional scale and short form-36 health survey questionnaire (SF-36) were used for data collection, followed by statistical analysis.

Results: Ambulatory status (as measured by the total lower extremity functional scale) revealed significant association $(\mathrm{P}<0.001)$ with age. The highest score belonged to the $10-30$ years age group. Also, there was a significant association $(\mathrm{P}=0.003)$ with marital status with the highest scores for unmarried ones. However, no significant $(\mathrm{P}=0.705)$ gender association was noted though scores were higher for the male gender. As regards, the social performance was measured by SF-36. The findings revealed a significant association $(\mathrm{P}<0.05)$ of most domains of SF-36 with age groups, with the highest scores for the age group of 10-30 years. Also, a significant association with the gender with higher scores in females was noted in most domains. In contrast, no significant association with marital status was reported in most domains.

Discussion: Ambulatory status has a significant association with age and marital status with no significant gender association. While social performance has a significant association with gender, most domains had significant associations with age groups. However, no association with marital status was present.

\footnotetext{
* Corresponding Author:

Hilal Ahmed, $P$ hD.

Address: Department of Otorhinolaryngology, Capital Hospital PGMI, Shaheed Zulfiqar Ali Bhutto University, Islamabad, Pakistan. Tel: +98 (33) 35101134

E-mail:ghulam_saqulain@yahoo.com
} 


\section{Highlights}

- Higher ambulatory status in transfemoral prosthesis users is associated with younger age group and single people.

- Better social performance in transfemoral prosthesis users is present in the younger age group and female gender.

- Marital status and gender have no association with social performance and ambulatory status for transfemoral prosthesis users, respectively.

\section{Plain Language Summary}

Amputation and, especially transfemoral, is a common surgical procedure requiring prosthetic use and rehabilitation to achieve the required ambulatory and social performance status. Since amputees suffer from physical and mental challenges, studying the determinants of prosthetic ambulation and the social performance of such amputees, along with an absence of related literature, was the stimulus for the study. This study was done to determine the association of ambulatory and social performance status of transfemoral prosthetic users with age, gender, and marital status. In this cross-sectional study, 400 transfemoral prosthesis users were studied using the lower extremity functional scale and 36-item short-form health survey questionnaire. The results revealed the association of ambulatory status with age and marital status with no significant gender association. The social performance showed an association with gender and age groups with no association with marital status.

\section{Introduction}

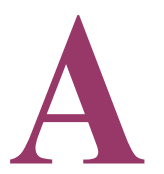

mputation is a common surgical procedure frequently needed in cases with trauma, diabetes mellitus, peripheral vascular diseases as well as neuropathy, accounting for 150000 cases of lower limb amputations per year in the United States alone [1], with an expected number of 3.6 million affected by 2050 [2]. Transfemoral is the second commonest amputation level found in a local study, with 20-40 years of the age group mainly being affected [3] and a higher prevalence $(77.6 \%)$ in males [4].

At the time and following the American Civil War (1961-1865), surgery involving amputations progressed with better survival of cases and the introduction of prosthetic devices, suspension technology, and improved material. The transfemoral amputation is the least desirable amputation and needs a prosthesis with socket, knee, pylon, and foot. It also requires a long rehabilitation period to improve physical activity, ambulation, and reintegration into daily life [5]. Rehabilitation success using prosthesis also depends on factors like caretaker time, device cost, rehab professionals, suitable fitting, and therapy facility [6].

Amputees suffer from many physical and mental challenges [7] and economic issues affecting their quality of life. Since they have no option but to accept new roles and coping strategies [8], ambulation and quality of life (QoL) should be the management's target.

Some strategies are used to assess the chances of prosthetic ambulation following major amputation of the lower limb; however, it is difficult to define the precise ambulatory function. Effective ambulation using a prosthesis has been noted as a prosthetic practice to achieve movement with or without external support. One study reported that only $46 \%$ of above-knee amputees achieve functional ambulation with a prosthesis [9].

Patients using prosthetic limbs should be assisted in a meaningful manner about how the rehabilitation interventions can improve their lives in relation to health in and out of their homes [10]. Following amputation, the patient puts on a prosthesis and needs to be properly followed rehabilitation to avoid deterioration of their physical activity and comorbidities affecting the overall health status of the amputee.

Following the lower extremity amputation and prosthetic fitting to optimize the outcome, it is desired to examine the predictive aspects as regards ambulation because this will help in developing the much needed clinical practice guidelines [11]. Some studies reveal that age and gender are partly associated with prosthetic ambulation [12]. 
Integration of lower limb prosthesis into body's selfimage is an essential part of rehabilitation; however, this is marred with an absence of knowledge of factors associated with prosthetic rehabilitation, especially those factors related to the psychological embodiment of the prosthesis [13]. Individuals with limited physical abilities suffer from lower QoL in the physical, environmental as well as psychological domains [14], including self-esteem. Therefore, further research is needed in the field and study factors associated with QoL of amputees using prostheses [15].

Hence, we conducted this study to determine the association of ambulatory and social performance status of transfemoral prosthetic users with their age, gender, and marital status. This study can be of immense help in topping up the deficient literature with no extensive research conducted on this topic in Pakistan. It also gives insight to researchers and clinicians for further studies and helps in clinical practice by determining factors associated with ambulatory status and QoL of transfemoral prosthetic users. It might also help provide insight to conduct investigations in the future to determine the factors associated with decreased functional status and social performance among prosthesis users.

\section{Materials and Methods}

This cross-sectional study recruited a sample of 400 transfemoral prosthesis users using the non-probability convenience sampling method from the Pakistan Institute of Prosthetic and Orthotic Sciences (PIPOS) over 6 months from July 1, 2019, to December 31, 2019. The sample included registered unilateral transfemoral prosthesis users of both genders, aged 10-60 years, who used the appliance for at least the last year. Orthosis users were excluded from the study. A sample size of 414 was calculated using the Raosoft online calculator with a 5\% margin of error, 20000 population size, $96 \%$ confidence level, and $50 \%$ response distribution. Then, 14 cases with incomplete data were excluded from the study leaving behind 400 cases which was the sample of our research.

A basic demographic sheet, the lower extremity functional scale (LEFS), and short form-36 health survey questionnaire (SF-36) were used for data collection. The study was carried out after obtaining ethical approval from the Institutional Research Board of ISRA University Islamabad, via approval No. 1709 M-Phil P\&O-005 dated June 17, 2019, followed by informed consent of the participants.
LEFS [16] is a valid 20-item scale to assess a person's ability to conduct everyday functions with unilateral or bilateral lower extremity disorder. Its maximum score is 80 , and the minimum clinically significant difference is 9 . The scale has a test-retest reliability of 0.94 .

SF-36 questionnaire is a valid questionnaire [17] that consists of 36 items to assess the QoL in eight dimensions of physical functioning, role limitations due to physical health issues, bodily pain, general health perceptions, vitality, social functioning, role limitations due to emotional issues, and mental health (psychological distress and wellbeing). It can be divided into physical component summary (PCS) and mental component summary (MCS). It has a composite score varying from 0 to 100 , with the average mean score of the general population being $50 \pm 10$, with a higher score showing a better QoL.

Data collection was done by the researcher himself from the sample population, which was obtained from cases registered at PIPOS. Sufficient time was given for the participant to respond to each item, and if necessary, the researcher explained the questions in the local language.

After data collection, they were analyzed in SPSS v. 21 . Continuous variables were presented as frequency and percentage, while categorical variables were presented as means and standard deviation. The Independent samples t-test and ANOVA were performed to analyze the association between study variables. P-values less than 0.05 were reported as significant.

\section{Results}

The current study revealed (Figure 1) a predominantly male population $(290[72.5 \%])$ with a majority in the age group of $10-30$ years $(155[38.8 \%])$ followed by $46-60$ years age group (153[38.3\%]) and the remaining in the age group of $31-45$ years. The majority of participants were married (250[62.5\%]), and the least was divorced (2[0.5\%]). Also, 188 patients $(47 \%)$ had normal BMI, and only $88(22 \%)$ were obese. Finally, trauma was the commonest (287[71.6\%]) cause of amputation (Figure 2).

The total score of LEFS revealed significant association $(\mathrm{P}<0.001)$ with age groups with the highest score $(57.79 \pm 13.06)$ for the age group of 10-30 years and lowest $(49.31 \pm 15.43)$ for the age group of $46-60$ years. Also, all LEFS items revealed a significant association with age with higher scores for younger age groups except item 6 (squatting), item 14 (standing for one hour), item 15 (sitting for one hour), and item 20 (rolling over in bed), which did not reveal significant associations with 


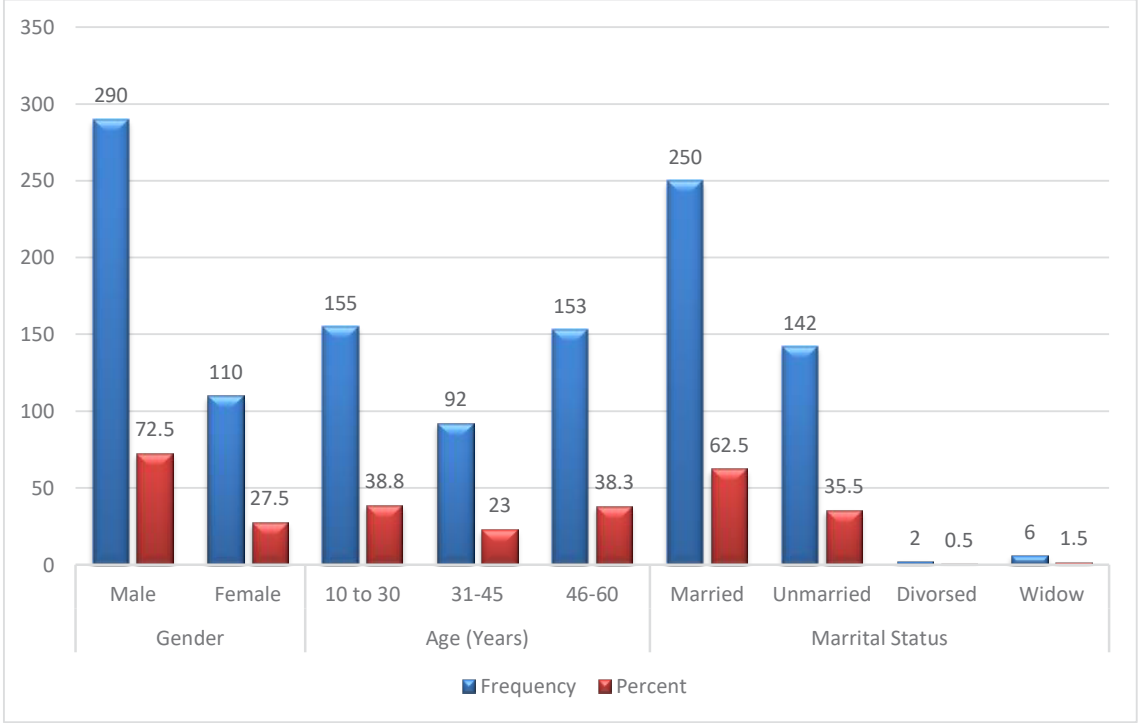

Figure 1. Demographic characteristics of the participants $(\mathrm{N}=400)$

Iranian Rehabilitation Journal

age (Table 1). As regards, SF-36 significant association of age groups was noted with the highest score for the younger age group of 10-30 years, for "physical function" (53.90 $\pm 16.86 ; \mathrm{P}<0.001)$, for "role limitation due to physical health" $(48.55 \pm 38.46 ; \mathrm{p}=0.002)$, for "energy" (57.10 $\pm 13.79 ; \mathrm{P}<0.001)$, for "emotional wellbeing" (60.23 $\pm 16.18 ; \mathrm{P}=0.037)$, for "pain" $(72.18 \pm 20.58$; $\mathrm{P}<0.001)$, and for "general health" $(57.68 \pm 13.41$; $\mathrm{P}<0.001)$. Although scores for the younger age group of 10 -30 years was highest $(49.81 \pm 39.21)$ for "limitation due to emotional health problem" and "social function" $(62.31 \pm 17.81)$, the differences were not significant with
$\mathrm{P}=0.687$ and $\mathrm{P}=0.412$, respectively. The results indicate higher quality of life in the younger age group.

Regarding the gender association (Table 2), the total score of LEFS did not reveal association $(\mathrm{P}=0.705)$ with participants' gender, though scores were higher for male gender $(54.26 \pm 15.69)$ compared to female (53.65 \pm 10.77$)$. However, few LEFS items revealed a significant association with the gender with higher scores for males, including squatting (1.97 \pm 0.92 ; $\mathrm{P}=0.001)$ and lifting objects from the floor $(2.33 \pm 0.90$; $\mathrm{P}<0.001)$. As regards, higher SF-36 scores were noted for females in most domains except physical function.

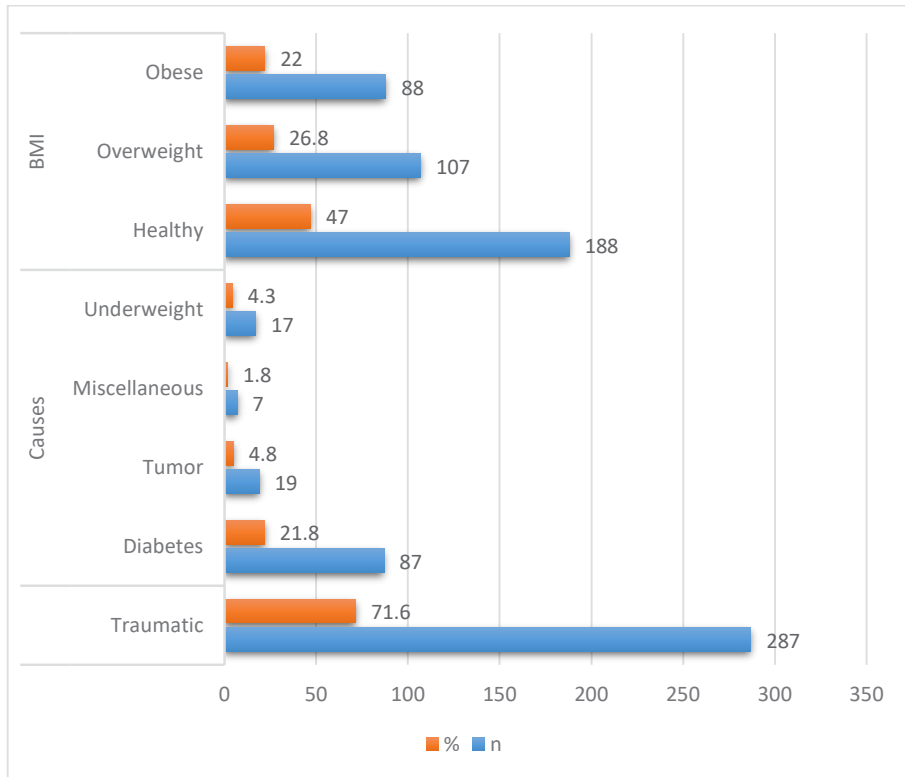

Figure 2. Body Mass Index and causes of amputation of the participants $(\mathrm{N}=400)$ 
Table 1. Lower extremity functional scale and short form-36 health survey questionnaire items versus age groups (cross tabulation and ANOVA statistics, $\mathrm{N}=400$ )

\begin{tabular}{|c|c|c|c|c|c|c|}
\hline \multirow{3}{*}{ Scale } & \multirow{3}{*}{ No. } & \multirow{3}{*}{ Item (Activities) } & \multirow{2}{*}{\multicolumn{3}{|c|}{$\begin{array}{c}\text { Mean } \pm S D \\
\text { Age Group (y) }\end{array}$}} & \multirow{3}{*}{$\begin{array}{c}\text { ANOVA Statistics } \\
\text { F, P }\end{array}$} \\
\hline & & & & & & \\
\hline & & & $\begin{array}{c}10-30 \\
155(38.75)\end{array}$ & $31-4592(23)$ & $46-60153(38.5)$ & \\
\hline \multirow{21}{*}{$\begin{array}{l}\text { Lower } \\
\text { Extrem- } \\
\text { ity Func- } \\
\text { tional } \\
\text { Scale }\end{array}$} & 1 & Usual work & $2.43 \pm 1.02$ & $2.11 \pm 1.07$ & $2.09 \pm 0.88$ & $5.498,0.004$ \\
\hline & 2 & Usual hobbies & $2.38 \pm 1.05$ & $2.04 \pm 1.07$ & $1.91 \pm 0.87$ & $9.185,0.001$ \\
\hline & 3 & $\begin{array}{l}\text { Getting into or out } \\
\text { of bath }\end{array}$ & $2.86 \pm 1.03$ & $2.48 \pm 1.11$ & $2.34 \pm 1.11$ & $9.598,0.001$ \\
\hline & 4 & $\begin{array}{l}\text { Walking between } \\
\text { rooms }\end{array}$ & $2.93 \pm 1.01$ & $2.54 \pm 1.17$ & $2.44 \pm 1.04$ & $8.692,0.001$ \\
\hline & 5 & $\begin{array}{l}\text { Putting on shoes or } \\
\text { socks }\end{array}$ & $2.59 \pm 1.01$ & $2.42 \pm 1.01$ & $2.29 \pm 0.92$ & $3.645,0.001$ \\
\hline & 6 & Squatting & $1.97 \pm 0.95$ & $1.90 \pm 0.95$ & $1.76 \pm 0.83$ & $2.130,0.120$ \\
\hline & 7 & $\begin{array}{l}\text { Lifting object from } \\
\text { floor }\end{array}$ & $2.41 \pm 0.90$ & $2.29 \pm 0.86$ & $2.03 \pm 0.87$ & $7.700,0.001$ \\
\hline & 8 & Light activities & $2.39 \pm 0.82$ & $2.52 \pm 0.75$ & $2.19 \pm 0.85$ & $5.122,0.006$ \\
\hline & 9 & Heavy activities & $2.24 \pm 0.85$ & $2.08 \pm 0.71$ & $1.89 \pm 0.90$ & $6.645,0.001$ \\
\hline & 10 & $\begin{array}{l}\text { Getting in and out of } \\
\text { the car }\end{array}$ & $2.50 \pm 0.96$ & $2.32 \pm 1.08$ & $2.14 \pm 1.03$ & $4.810,0.009$ \\
\hline & 11 & Walking two blocks & $2.29 \pm 0.87$ & $2.26 \pm 0.85$ & $2.05 \pm 0.98$ & $3.166,0.043$ \\
\hline & 12 & Walking a mile & $2.19 \pm 0.88$ & $2.30 \pm 0.86$ & $1.81 \pm 0.89$ & $11.25,0.001$ \\
\hline & 13 & $\begin{array}{l}\text { Climbing ten stairs up } \\
\text { or down }\end{array}$ & $2.21 \pm 0.90$ & $2.15 \pm 0.86$ & $1.78 \pm 0.88$ & $10.35,0.001$ \\
\hline & 14 & Standing one hour & $2.25 \pm 0.87$ & $2.15 \pm 0.86$ & $2.00 \pm 0.95$ & $2.884,0.057$ \\
\hline & 15 & Sitting for one hour & $2.53 \pm 1.07$ & $2.50 \pm 0.99$ & $2.27 \pm 1.16$ & $2.521,0.082$ \\
\hline & 16 & $\begin{array}{l}\text { Running on even } \\
\text { ground }\end{array}$ & $2.12 \pm 0.96$ & $2.09 \pm 0.87$ & $1.63 \pm 1.02$ & $11.51,0.001$ \\
\hline & 17 & $\begin{array}{l}\text { Running on uneven } \\
\text { ground }\end{array}$ & $1.81 \pm 0.89$ & $1.98 \pm 0.85$ & $1.46 \pm 0.97$ & $10.85,0.001$ \\
\hline & 18 & $\begin{array}{l}\text { Running with sharp } \\
\text { turns }\end{array}$ & $1.94 \pm 0.96$ & $2.09 \pm 1.00$ & $1.52 \pm 0.99$ & $11.68,0.001$ \\
\hline & 19 & Hopping & $1.86 \pm 1.00$ & $2.13 \pm 1.03$ & $1.48 \pm 1.02$ & $12.844,0.001$ \\
\hline & \multirow[t]{2}{*}{20} & Rolling in bed & $2.32 \pm 1.09$ & $2.30 \pm 1.22$ & $2.22 \pm 1.18$ & $0.364,0.695$ \\
\hline & & Total score & $57.79 \pm 13.06$ & $55.82 \pm 13.01$ & $49.31 \pm 15.43$ & $15.00,0.001$ \\
\hline \multirow{8}{*}{$\begin{array}{c}\text { Short } \\
\text { Form-36 } \\
\text { Health } \\
\text { Survey } \\
\text { Ques- } \\
\text { tion- } \\
\text { naire }\end{array}$} & 1 & Physical function & $53.90 \pm 16.86$ & $53.74 \pm 17.40$ & $42.42 \pm 23.98$ & $15.38,0.001$ \\
\hline & 2 & $\begin{array}{l}\text { Role limitation due to } \\
\text { physical health } \\
\text { Limitation due to }\end{array}$ & $48.55 \pm 38.46$ & $43.68 \pm 41.43$ & $32.84 \pm 37.44$ & $6.52,0.002$ \\
\hline & 3 & $\begin{array}{l}\text { emotional health } \\
\text { problem }\end{array}$ & $49.81 \pm 39.21$ & $48.39 \pm 40.41$ & $45.86 \pm 41.33$ & $0.37,0.687$ \\
\hline & 4 & Energy/Fatigue & $57.10 \pm 13.79$ & $52.86 \pm 13.91$ & $49.90 \pm 13.52$ & $10.68,0.001$ \\
\hline & 5 & Emotional wellbeing & $60.23 \pm 16.18$ & $57.51 \pm 16.94$ & $55.57 \pm 14.97$ & $3.33,0.037$ \\
\hline & 6 & Social function & $62.31 \pm 17.81$ & $62.09 \pm 14.25$ & $59.97 \pm 16.38$ & $0.89,0.412$ \\
\hline & 7 & Pain & $72.18 \pm 20.58$ & $68.57 \pm 19.08$ & $62.99 \pm 17.75$ & $8.91,0.001$ \\
\hline & 8 & General Health & $57.68 \pm 13.41$ & $52.09 \pm 13.79$ & $46.36 \pm 13.86$ & $26.39,0.001$ \\
\hline
\end{tabular}

Data are presented as No.(\%) or Mean \pm SD. ${ }^{*} \mathrm{P}<0.05,{ }^{* *} \mathrm{P}<0.001$. 
Table 2. Lower Extremity Functional Scale and Short Form-36 Health Survey Questionnaire Items Versus Gender (Cross Tabulation and t-test Statistics, $\mathrm{n}=400$ )

\begin{tabular}{|c|c|c|c|c|c|}
\hline \multirow{3}{*}{ Scale } & \multirow{3}{*}{ No. } & \multirow{3}{*}{ Items (Activities) } & \multicolumn{2}{|c|}{ Mean $\pm S D$} & \multirow{3}{*}{$\begin{array}{l}\text { t-test } \\
t, P\end{array}$} \\
\hline & & & \multicolumn{2}{|c|}{ Gender } & \\
\hline & & & Male, 290(72.5) & Female, 110(27.5) & \\
\hline \multirow{21}{*}{$\begin{array}{l}\text { Lower Extrem- } \\
\text { ity Functional } \\
\text { Scale }\end{array}$} & 1 & Usual work & $2.25 \pm 0.98$ & $2.17 \pm 1.03$ & $0.678,0.498$ \\
\hline & 2 & Usual hobbies & $2.17 \pm 1.03$ & $1.99 \pm 0.93$ & $1.612,0.108$ \\
\hline & 3 & Getting into or out of the bath & $2.48 \pm 1.05$ & $2.82 \pm 1.19$ & $-2.74,0.006$ \\
\hline & 4 & Waling between rooms & $2.58 \pm 1.02$ & $2.85 \pm 1.20$ & $-2.79,0.023$ \\
\hline & 5 & Putting on shoes or socks & $2.45 \pm 0.97$ & $2.42 \pm 1.01$ & $0.273,0.785$ \\
\hline & 6 & Squatting & $1.97 \pm 0.92$ & $1.62 \pm 0.83$ & $3.501,0.001$ \\
\hline & 7 & Lifting object from the floor & $2.33 \pm 0.90$ & $1.98 \pm 0.82$ & $3.477,0.001$ \\
\hline & 8 & Light activities & $2.37 \pm 0.83$ & $2.27 \pm 0.81$ & $1.041,0.299$ \\
\hline & 9 & Heavy activities & $2.10 \pm 0.88$ & $1.97 \pm 0.77$ & $1.368,0.172$ \\
\hline & 10 & Getting in and out of the car & $2.32 \pm 0.99$ & $2.31 \pm 1.12$ & $0.101,0.920$ \\
\hline & 11 & Walking two blocks & $2.23 \pm 0.91$ & $2.08 \pm 0.90$ & $1.464,0.144$ \\
\hline & 12 & Walking a mile & $2.10 \pm 0.95$ & $1.99 \pm 0.77$ & $1.078,0.282$ \\
\hline & 13 & Going ten stairs up or down & $2.07 \pm 0.89$ & $1.93 \pm 0.94$ & $1.430,0.153$ \\
\hline & 14 & Standing one hour & $2.15 \pm 0.93$ & $2.07 \pm 0.83$ & $0.779,0.437$ \\
\hline & 15 & Sitting for one hour & $2.37 \pm 1.08$ & $2.56 \pm 1.11$ & $-1.595,0.111$ \\
\hline & 16 & Running on even ground & $1.87 \pm 1.00$ & $2.07 \pm 0.95$ & $-1.812,0.071$ \\
\hline & 17 & Running on uneven ground & $1.70 \pm 1.00$ & $1.75 \pm 0.74$ & $-0.521,0.602$ \\
\hline & 18 & Running with sharp turns & $1.78 \pm 1.03$ & $1.92 \pm 0.93$ & $-1.267,0.206$ \\
\hline & 19 & Hopping & $1.78 \pm 1.07$ & $1.77 \pm 0.96$ & $0.056,0.955$ \\
\hline & \multirow[t]{2}{*}{20} & Rolling in bed & $2.24 \pm 1.19$ & $2.36 \pm 1.05$ & $-0.922,0.357$ \\
\hline & & Total score & $54.26 \pm 15.69$ & $53.65 \pm 10.77$ & $0.379,0.705$ \\
\hline \multirow{8}{*}{$\begin{array}{l}\text { Short Form-36 } \\
\text { Health Survey } \\
\text { Questionnaire }\end{array}$} & 1 & Physical function & $50.11 \pm 21.74$ & $47.75 \pm 17.69$ & $1.018,0.309$ \\
\hline & 2 & $\begin{array}{l}\text { Role limitation due to physical } \\
\text { health }\end{array}$ & $38.93 \pm 38.62$ & $47.95 \pm 40.51$ & $-2.058,0.040$ \\
\hline & 3 & $\begin{array}{l}\text { Limitation due to emotional health } \\
\text { problem }\end{array}$ & $47.66 \pm 40.76$ & $48.79 \pm 39.02$ & $-0.250,0.803$ \\
\hline & 4 & Energy/Fatigue & $52.15 \pm 14.03$ & $56.59 \pm 13.63$ & $-2.851,0.005$ \\
\hline & 5 & Emotional wellbeing & $56.21 \pm 15.40$ & $62.05 \pm 16.82$ & $-3.30,0.001$ \\
\hline & 6 & Social Function & $60.10 \pm 16.66$ & $64.66 \pm 15.69$ & $-2.48,0.014$ \\
\hline & 7 & Pain & $65.98 \pm 19.04$ & $72.70 \pm 20.17$ & $-3.10,0.002$ \\
\hline & 8 & General Health & $51.17 \pm 14.46$ & $54.41 \pm 14.47$ & $-2.0,0.046$ \\
\hline
\end{tabular}

Data are presented as No.(\%) or Mean \pm SD. ${ }^{*} \mathrm{P}<0.05,{ }^{* *} \mathrm{P}<0.001$.

Iranian Rehabilitation Journal 
Table 3. Lower extremity functional scale and short form-36 health survey questionnaire items versus marital status (cross tabulation and ANOVA statistics, $\mathrm{N}=400$ )

\begin{tabular}{|c|c|c|c|c|c|c|}
\hline \multirow{3}{*}{ Scale } & \multirow{3}{*}{ Item activities } & \multicolumn{4}{|c|}{ Mean \pm SD } & \multirow{3}{*}{$\begin{array}{l}\text { ANOVA Statis- } \\
\text { tics F,P-value }\end{array}$} \\
\hline & & \multicolumn{4}{|c|}{ Marital Status (n) } & \\
\hline & & $\begin{array}{l}\text { Married } \\
\text { 250(62.5) }\end{array}$ & $\begin{array}{l}\text { Unmarried } \\
142(25.5)\end{array}$ & Divorced 2(0.5) & Widow 6(1.5) & \\
\hline \multirow{21}{*}{$\begin{array}{l}\text { Lower } \\
\text { Extrem- } \\
\text { ity } \\
\text { Func- } \\
\text { tional } \\
\text { Scale }\end{array}$} & Usual work & $2.18 \pm 0.96$ & $2.32 \pm 1.06$ & $2.00 \pm 1.41$ & $1.83 \pm 0.75$ & $0.954,0.414$ \\
\hline & Usual hobbies & $2.04 \pm 0.96$ & $2.30 \pm 1.06$ & $2.00 \pm 1.41$ & $1.33 \pm 0.82$ & $3.37,0.019$ \\
\hline & Getting into or out of bath & $2.54 \pm 1.08$ & $2.68 \pm 1.13$ & $3.00 \pm 1.41$ & $1.67 \pm 0.52$ & $1.98,0.117$ \\
\hline & Walking between rooms & $2.63 \pm 1.09$ & $2.73 \pm 1.07$ & $2.50 \pm 0.71$ & $2.17 \pm 0.98$ & $0.672,0.569$ \\
\hline & Putting on shoes or socks & $2.42 \pm 0.96$ & $2.51 \pm 1.02$ & $3.00 \pm 0.00$ & $1.67 \pm 0.52$ & $1.74,0.159$ \\
\hline & Squatting & $1.79 \pm 0.85$ & $2.01 \pm 0.99$ & $2.00 \pm 0.00$ & $2.17 \pm 0.98$ & $2.00,0.113$ \\
\hline & Lifting object from the floor & $2.19 \pm 0.88$ & $2.34 \pm 0.92$ & $2.50 \pm 0.71$ & $1.83 \pm 0.75$ & $1.32,0.266$ \\
\hline & Light activities & $2.32 \pm 0.82$ & $2.41 \pm 0.84$ & $2.00 \pm 0.00$ & $1.83 \pm 0.75$ & $1.24,0.294$ \\
\hline & Heavy activities & $1.96 \pm 0.82$ & $2.23 \pm 0.86$ & $3.00 \pm 0.00$ & $2.33 \pm 1.51$ & $4.17,0.006$ \\
\hline & $\begin{array}{l}\text { Getting in and out of the } \\
\text { car }\end{array}$ & $2.26 \pm 1.05$ & $2.44 \pm 0.96$ & $2.50 \pm 0.71$ & $1.67 \pm 1.51$ & $1.81,0.144$ \\
\hline & Walking two blocks & $2.14 \pm 0.89$ & $2.29 \pm 0.91$ & $3.50 \pm 0.71$ & $1.67 \pm 1.51$ & $2.92,0.034$ \\
\hline & Walking a mile & $2.00 \pm 0.88$ & $2.21 \pm 0.91$ & $2.00 \pm 0.00$ & $1.50 \pm 1.22$ & $2.42,0.065$ \\
\hline & Going ten stairs up or down & $1.93 \pm 0.88$ & $2.22 \pm 0.90$ & $2.00 \pm 0.00$ & $2.00 \pm 1.55$ & $3.14,0.025$ \\
\hline & Standing one hour & $2.06 \pm 0.90$ & $2.25 \pm 0.88$ & $3.50 \pm 0.71$ & $1.50 \pm 1.22$ & $3.90,0.009$ \\
\hline & Sitting for one hour & $2.38 \pm 1.12$ & $2.54 \pm 1.03$ & $2.50 \pm 0.71$ & $1.50 \pm 1.22$ & $2.08,0.102$ \\
\hline & Running on even ground & $1.81 \pm 0.96$ & $2.14 \pm 0.99$ & $3.00 \pm 1.41$ & $1.50 \pm 1.22$ & $4.69,0.003$ \\
\hline & Running on uneven ground & $1.64 \pm 0.96$ & $1.87 \pm 0.87$ & $2.50 \pm 0.71$ & $0.83 \pm 0.75$ & $4.07,0.007$ \\
\hline & Running with sharp turns & $1.74 \pm 1.01$ & $1.95 \pm 0.98$ & $3.00 \pm 0.00$ & $1.33 \pm 1.03$ & $2.75,0.042$ \\
\hline & Hopping & $1.68 \pm 1.01$ & $1.96 \pm 1.06$ & $2.50 \pm 0.71$ & $1.17 \pm 1.47$ & $3.13,0.026$ \\
\hline & Rolling in bed & $2.26 \pm 1.15$ & $2.30 \pm 1.14$ & $2.50 \pm 0.71$ & $2.17 \pm 1.72$ & $0.077,0.972$ \\
\hline & Total score & $52.59 \pm 14.29$ & $57.11 \pm 13.73$ & $64.38 \pm 9.72$ & $42.08 \pm 25.72$ & $4.80,0.003$ \\
\hline \multirow{8}{*}{$\begin{array}{c}\text { Short } \\
\text { Form-36 } \\
\text { Health } \\
\text { Survey } \\
\text { Ques- } \\
\text { tion- } \\
\text { naire }\end{array}$} & Physical function & $46.43 \pm 21.04$ & $54.68 \pm 18.70$ & $45.00 \pm 7.07$ & $53.33 \pm 32.35$ & $5.04,0.002$ \\
\hline & $\begin{array}{l}\text { Role limitation due to physi- } \\
\text { cal health }\end{array}$ & $41.47 \pm 40.78$ & $41.73 \pm 37.16$ & $12.50 \pm 17.68$ & $41.67 \pm 34.16$ & $0.362,0.780$ \\
\hline & $\begin{array}{l}\text { Limitation due to emotional } \\
\text { health problem }\end{array}$ & $49.08 \pm 40.69$ & $45.21 \pm 39.59$ & $50.00 \pm 23.57$ & $66.67 \pm 42.16$ & $0.717,0.542$ \\
\hline & Energy/Fatigue & $52.03 \pm 14.32$ & $55.63 \pm 13.55$ & $57.50 \pm 17.68$ & $54.17 \pm 6.65$ & $2.07,0.104$ \\
\hline & Emotional wellbeing & $58.08 \pm 16.17$ & $57.55 \pm 16.09$ & $56.00 \pm 16.97$ & $54.00 \pm 5.51$ & $0.158,0.925$ \\
\hline & Social function & $62.07 \pm 16.15$ & $60.09 \pm 17.31$ & $53.75 \pm 12.37$ & $64.58 \pm 12.29$ & $0.650,0.583$ \\
\hline & Pain & $67.20 \pm 19.31$ & $69.37 \pm 20.30$ & $55.00 \pm 0.00$ & $62.08 \pm 13.08$ & $0.836,0.475$ \\
\hline & General Health & $50.39 \pm 14.90$ & $55.07 \pm 13.70$ & $52.50 \pm 3.54$ & $50.00 \pm 8.37$ & $3.22,0.023$ \\
\hline
\end{tabular}

Data are presented as No. $(\%)$ or Mean \pm SD. ${ }^{*} \mathrm{P}<0.05,{ }^{* *} \mathrm{P}<0.001$. 
However, a significant association with gender was noted for "role limitation due to physical health" $(\mathrm{P}=0.040)$, “energy" $(\mathrm{P}=0.005)$, "emotional wellbeing" $(\mathrm{P}=0.001)$, "social function" $(\mathrm{P}=0.014)$, "pain" $(\mathrm{P}=0.002)$, and "general health" $(\mathrm{P}=0.046)$. Regarding the association with marital status (Table 3), the total score of LEFS revealed a significant association $(\mathrm{P}=0.003)$ with the marital status of the participants with the highest LEFS score for unmarried $(57.11 \pm 13.73)$ and lowest for widows (42.08 \pm 25.72$)$. Also, around half of LEFS items revealed significant associations. These items were usual hobbies $(\mathrm{P}=0.019)$, heavy activities $(\mathrm{P}=0.006)$, walking two blocks $(\mathrm{P}=0.034)$, climbing ten stairs up or down $(\mathrm{P}=0.025)$, standing one hour $(\mathrm{P}=0.009)$, running on even ground $(\mathrm{P}=0.003)$, running on uneven ground $(\mathrm{P}=0.007)$, running with sharp turns $(\mathrm{P}=0.042)$, and hopping $(\mathrm{P}=0.026)$. In contrast, other items did not reveal a significant association. On the other hand, SF-36 domain scores showed no significant association with marital status except for domains of physical function $(\mathrm{P}=0.002)$ and general health $(\mathrm{P}=0.023)$, with the highest scores for unmarried participants.

\section{Discussion}

This study aimed to determine the association of ambulatory and social performance status of transfemoral prosthetic users with their gender, age, and marital status. A total of 400 transfemoral amputees using prosthetic devices were studied. The sample included a predominantly male population $(72.5 \%)$ since men are more exposed to trauma. This finding is consistent with another study in which the male population dominated $(66 \%)$ [18]. The current study did not reveal any significant association $(\mathrm{P}=0.705)$ of the total score of LEFS with participants' gender, though the score was higher in males. However, few LEFS items revealed a significant association with the gender with higher scores for males, including squatting $(\mathrm{P}=0.001)$ and lifting objects from the floor $(\mathrm{P}<0.001)$. This result might be because males are usually more muscular and stronger. While Kahle JT et al., in their review study, noted that gender was a minimal support factor while considering the candidacy of prosthetic use [12].

Regarding social performance, the higher mean SF-36 scores were noted for females in most domains except physical function. However, a significant association with gender was noted for role limitation due to physical health $(\mathrm{P}=0.040)$, energy $(\mathrm{P}=0.005)$, emotional wellbeing $(\mathrm{P}=0.001)$, social function $(\mathrm{P}=0.014)$, pain $(\mathrm{P}=0.002)$, and general health $(\mathrm{P}=0.046)$. In contrast, Knežević A et al. [19], Pran L et al. [20], and Sinha R et al. [7] studies did not reveal a significant association of SF-36 scores with gender. While another local study by Kamran et al. [15] showed a significant association of stress $(\mathrm{P}=0.28)$ and anxiety (0.029) with gender, no association of depression and self-esteem was noted. It is important to note that lower social performance in men might result from their worries about catering to their families [8]. They are the members who go out for earning and meet other family requirements in eastern culture. In contrast, a study by Matos et al. [21] revealed the male gender has comparatively better QoL than females who had lower performance in all domains of SF-36.

In the current study, most cases were in the age groups of 12-30 and 46-60 years, and the ambulatory function had a significant association $(\mathrm{P}<0.001)$ with age with the highest scores for the age group of 10-30 years and lowest for the age group of 46-60 years. Regarding the LEFS items, significant association with age was seen, with higher scores for the younger age groups except item 6 (squatting), item 14 (stand for one hour), item 15 (sit for one hour), and item 20 (rolling over in bed) indicating that somehow these activities were not related with age. According to Wurdeman SR et al. [6], a patient's age affects mobility when comparing $<65$ years old people with $>65$ years age group with reduced ambulation in elderly. In a systematic review, Kahle JT et al. [12] noted that increasing age of amputees was associated with less ambulation using prosthetic devices; hence age was a strong predictive factor for the candidacy of the prosthetic device. Good ambulatory function as represented by LEFS mean score was reported in another local study in the younger population with a mean age of 30 years with the mean LEFS of 49.40 at the $4^{\text {th }}$ week and 59.27 at the $12^{\text {th }}$ week [22].

In the present study, the social performance showed a significant association with age with the highest score for the younger age group of 10-30 years, for "physical function" $(\mathrm{P}<0.001)$, for "the role limitation due to physical health" $(\mathrm{P}=0.002)$, for "energy" $(\mathrm{P}<0.001)$, for "emotional wellbeing" ( $\mathrm{P}=-0.037)$, for "pain" $(\mathrm{P}<0.001)$, and for "general health" $(\mathrm{P}<0.001)$. These results are probably due to better physical state and because age and time elapsed since the procedure were responsible for 3\% variation in PCS scores of SF-36 [7]. Although scores for the younger age group of 10-30 years were highest for "limitation due to emotional health problem" and "social function", the difference was not significant. Hence higher QoL, including social performance, was seen at a younger age. Significant association of QoL was noted with age group when increasing age is related to more problems and poor quality in a Caribbean study 
[20]. Sinha $\mathrm{R}$ et al. have also reported a significant association of age with PCS and MCS scores of SF-36 [7]. In contrast, a local study revealed a significant association of depression ( $\mathrm{P}=0.001)$ with the age group of patients, while no significant association of stress, anxiety, and self-esteem was seen [15].

In the current study, the majority of the population was married (250[62.5\%]) and marital status revealed a significant association ( $\mathrm{P}=0.003$ ) with ambulatory function as represented by the total score of LEFS, with the highest LEFS score for unmarried with almost half of LEFS items having significant associations $(\mathrm{P}<0.05)$ with marital status, including usual hobbies, heavy activities, walking two blocks, climbing ten stairs up or down, standing one hour, running on even ground, running on uneven ground, running with sharp turns, and hopping. On the other hand, in the current study, social performance as assessed by SF-36 domain scores did not reveal any significant association with marital status except for domains of physical function and general health. These domains showed significant association with the highest scores for unmarried participants. A local study revealed a significant association of depression $(\mathrm{P}=0.008)$ and self-esteem $(\mathrm{P}=0.011)$ with the marital status of the individual, while no association of stress and anxiety was noted [15]. Another study reported that family life was affected in 16 cases $(32 \%)$ while social life was involved in 10 cases $(20 \%)$, indicating the importance of prosthesis in QoL [18]. According to Amoah VMK et al., catering to the amputee's family's needs was an issue for which male amputees were worried [8].

Our study was conducted in a few areas of Pakistan and did not cater to the variety of transfemoral prostheses. Hence its results cannot be generalized. Another limitation was the lack of control over confounding variables. We recommend future trials using different prosthetic devices.

\section{Conclusion}

Ambulatory status has a significant association with age and marital status with no significant association with gender. Social performance has a significant association with gender, and most domains have significant associations with age groups. However, no association with marital status was found.

\section{Ethical Considerations}

\section{Compliance with ethical guidelines}

The study was approved by Institutional Research Board of ISRA University Islamabad, vide Reg \# 1709 M-Phil P\&O-005.

\section{Funding}

This research did not receive any grant from funding agencies in the public, commercial, or non-profit sectors.

\section{Authors' contributions}

Conceptualization \& supervision: Shafiq ur Rehman, Aqeel Ahmed Khan; Methodology, Resources \& data curation: Shafiq ur Rehman, Muhammad Kamran; Formal analysis and writing-original draft preparation: Muhammad Kamran, Hilal Ahmed; Writing - review \& editing \& supervision: Ghulam Saqulain, Aqeel Ahmed Khan.

\section{Conflict of interest}

The authors declared no conflict of interest.

\section{Reference}

[1] Molina CS, Faulk JB. Lower Extremity Amputation. In: StatPearls [Internet]. Treasure Island (FL): StatPearls Publishing 2021 Jan. [PMID]

[2] Ziegler-Graham K, MacKenzie EJ, Ephraim PL, Travison TG, Brookmeyer R. Estimating the prevalence of limb loss in the United States: 2005 to 2050. Archives of Physical Medicine and Rehabilitation. 2008; 89(3):422-9. [DOI:10.1016/j. apmr.2007.11.005] [PMID]

[3] Rathore FA, Ayaz SB, Mansoor SN, Qureshi AR, Fahim M Demographics of lower limb amputations in the Pakistan military: A single center, three-year prospective survey. Cureus. 2016; 8(4):e566. [DOI:10.7759/cureus.566]

[4] Soomro N, Khan M, Ahmed SI, Minhas MA. Determinants of lower extremity amputations: An institutional experience. Journal of College of Physicians and Surgeons Pakistan 2013; 23(7):491-4. [PMID]

[5] O'Keeffe B, Rout S. Prosthetic rehabilitation in the lower limb. Indian Journal of Plastic Surgery. 2019; 52(1):134-43. [DOI:10.1055/s-0039-1687919] [PMID] [PMCID]

[6] Wurdeman SR, Stevens PM, Campbell JH. Mobility analysis of amputees II: Comorbidities and mobility in lower limb prosthesis users. American Journal of Physical Medicine \& Rehabilitation. 2018; 97(11):782-8. [DOI:10.1097/ PHM.0000000000000967] [PMID] [PMCID] 
[7] Sinha R, van den Heuvel WJ, Arokiasamy P. Factors affecting quality of life in lower limb amputees. Prosthetics and Orthotics International. 2011; 35(1):90-6. [DOI:10.1177/0309364610397087] [PMID]

[8] Amoah VMK, Anokye R, Acheampong E, Dadson R, Osei M, Nadutey A. The experiences of people with diabetesrelated lower limb amputation at the Komfo Anokye Teaching Hospital (KATH) in Ghana. BMC Research Notes. 2018, 11(1):66. [DOI:10.1186/s13104-018-3176-1] [PMID] [PMCID]

[9] Moore TJ, Barron JE, Golden C, Ellis C, Humphries D. Prosthetic usage following major lower extremity amputation. Clinical Orthopaedics and Related Research. 1989; (238):219-24 [DOI:10.1097/00003086-198901000-00032]

[10] Schaffalitzky E, Gallagher P, Maclachlan M, Ryall N. Understanding the benefits of prosthetic prescription: Exploring the experiences of practitioners and lower limb prosthetic users. Disability and Rehabilitation. 2011; 33(15-16):1314-23. [DOI:10.3109/09638288.2010.529234] [PMID]

[11] Dobson A, El-Gamil A, Shimer M, DaVanzo JE. Economic value of prosthetic services among medicare beneficiaries: A claimsbased retrospective cohort study. Military Medicine. 2016; 181(S 2):18-24. [DOI:10.7205/MILMED-D-15-00545] [PMID]

[12] Kahle JT, Highsmith MJ, Schaepper H, Johannesson A, Orendurff MS, Kaufman K. Predicting walking ability following lower limb amputation: An updated systematic literature review. Technology and Innovation. 2016; 18(2-3):125-37. [DOI:10.21300/18.2-3.2016.125] [PMID] [PMCID]

[13] Bekrater-Bodmann R. Factors associated with prosthesis embodiment and its importance for prosthetic satisfaction in lower limb amputees. Frontiers in Neurorobotics. 2021 14:604376. [DOI:10.3389/fnbot.2020.604376] [PMID] [PMCID]

[14] Magnusson L, Ghosh R, Jensen KR, Göbel K, Wågberg J, Wallén S, et al. Quality of life of prosthetic and orthotic users in South India: A cross-sectional study. Health and Quality of Life Outcomes. 2019; 17(1):50. [DOI:10.1186/s12955-0191116-y] [PMID] [PMCID]

[15] Kamran M, Mumtaz N, Saqulain G. Amalgamation of selfesteem, depression, anxiety and stress among prosthesis users. Journal of Pakistan Medical Association. 2021; 71(3):834-7. [DOI:10.47391/JPMA.872]

[16] Mehta SP, Fulton A, Quach C, Thistle M, Toledo C, Evans NA. Measurement properties of the lower extremity functional scale: A systematic review. Journal of Orthopaedic \& Sports Physical Therapy. 2016; 46(3):200-16. [DOI:10.2519/ jospt.2016.6165] [PMID]

[17] Zhang Y, Qu B, Lun Ss, Guo Y, Liu J. The 36-item short form health survey: Reliability and validity in Chinese medical students. International Journal of Medical Sciences. 2012; 9(7):521-6. [DOI:10.7150/ijms.4503] [PMID] [PMCID]

[18] Amjad T, Umay Kalsoom UK, Bairam S. Quality of life among lower limb prosthesis users attending artificial limb center of fauji foundation hospital Rawalpindi. Pakistan Armed Forces Medical Journal. 2018; 68(1):114-8. https:// www.pafmj.org/index.php/PAFMJ/article/view/1576

[19] Knežević A, Salamon T, Milankov M, Ninković S, Jeremić Knežević M, Tomašević Todorović $S$. Assessment of quality of life in patients after lower limb amputation. Medicinski Pregled. 2015; 68(3-4):103-8. [DOI:10.2298/MPNS1504103K] [PMID]
[20] Pran L, Baijoo S, Cave C, Slim H, Hamanan D, Maharaj R et al. A Caribbean perspective: Quality of life in major lower extremity amputees. 2020 [DOI:10.21203/rs.3.rs-54128/v1]]

[21] Matos DR, Naves JF, Araujo TCCF. Quality of life of patients with lower limb amputation with prostheses. Estudos de Psicologia (Campinas). 2020; 37:e190047. [DOI:10.1590/19820275202037e190047]

[22] Gozaydinoglu S, Hosbay Z, Durmaz H. Body image perception, compliance with a prosthesis and cognitive performance in transfemoral amputees. Acta Orthopaedica et Traumatologica Turcica. 2019; 53(3):221-5. [DOI:10.1016/j. aott.2019.03.014] [PMID] [PMCID] 